\title{
Resonant inelastic x-ray scattering (RIXS) spectra of magnesium diboride
}

\author{
K. Kokko*, V. Kulmala, and J.A. Leiro \\ Department of Physics, University of Turku, FIN-20014 Turku, Finland \\ W. Hergert \\ Department of Physics, Martin-Luther-University, \\ Friedemann-Bach-Platz 6, D-06099 Halle, Germany
}

(Dated: November 20, 2018)

\begin{abstract}
Using the tight-binding linear muffin-tin orbitals method, the soft x-ray fluorescence K-emission spectra of boron in $\mathrm{MgB}_{2}$, excited close to the absorption edge, are estimated. In the calculations the angle of incidence $\theta$ between the direction of the incoming photon and the hexagonal axis of the specimen is $60^{\circ}$ and $75^{\circ}$. Comparison with experiment is possible in the former case where good agreement is found. Furthermore, another resonant feature below the Fermi energy is predicted for the larger angle. This feature can be related to the excitations to the antibonding $\mathrm{B} \pi$-band in the neighbourhood of the $\mathrm{L}-\mathrm{H}$ line in the Brillouin zone.
\end{abstract}

PACS numbers: 71.20-b, 74.25.Jb, 78.70.Ck, 78.70.En

Magnesium diboride is a new kind of superconductor having a critical temperature of $39 \mathrm{~K}[\mathbf{1}]$. Although it was earlier widely used in chemical technology, the superconducting property of this compound was not discovered until now [2]. At the moment, $\mathrm{MgB}_{2}$ has attracted considerable attention regarding spectroscopic investigations [3]. For instance, angle resolved photoemission from single crystals have revealed clear dispersions of the occupied valence bands [4]. Additional information has been obtained using x-ray absorption - (XAS) and emission spectroscopy (XES) [5] . In x-ray K-absorption a corehole is created for the 1s level by a photon and the resulting photoelectron is escaping from the specimen. The K-emission band can be measured when the deep lying level will be filled by a valence-electron and another photon is emitted. The resonant inelastic scattering (RIXS) differs from the conventional XES in the sense that the photoelectron remains in the conduction band above the Fermi surface and it will be absorbed by the sample [6].

Utilizing synchrotron radiation, resonant inelastic $\mathrm{x}$ ray scattering is a promising method to probe elementspecific, local momentum-resolved electronic structure of systems that are difficult to investigate using other techniques. The symmetry of the occupied and unoccupied states is coupled with the polarization and direction of the incoming and outgoing radiation. Crystal momentum conservation has been observed in many materials e.g. in hexagonal boron nitride and graphite [7]. Theoretical consideration of the RIXS within the band structure picture is given e.g. in [8].

In RIXS, a photon $\left(\hbar \omega_{q}\right)$ comes to the sample, excites a core level $\left(\epsilon_{a}\right)$ electron to the conduction state $\left(\epsilon_{c}\right)$ and a valence electron $\left(\epsilon_{v}\right)$ drops to the core hole emitting a photon $\left(\hbar \omega_{q^{\prime}}\right)$. The formula for the doubly differential cross section in the dipole approximation (the wave vectors of the ingoing and outgoing photons are small, $q \approx q^{\prime} \approx 0$, compared to the dimensions of the Brillouin zone of the Bloch states $(\vec{k})$ of the electrons) implemented in our x-ray spectrum program (XSPEC) 9] is

$$
\begin{aligned}
\frac{d^{2} \sigma}{d \Omega d\left(\hbar \omega_{q^{\prime}}\right)} \propto & \frac{\omega_{q^{\prime}}}{\omega_{q}} \sum_{b_{c}, b_{v}, \vec{k}, m_{s}}\left|\sum_{t, m_{j}} M_{t, m_{j}, m_{s}}^{b_{v}, \vec{k}} M_{t, m_{j}, m_{s}}^{* b_{b}, \vec{k}}\right|^{2} \\
& \times \delta\left(\epsilon_{a}-\epsilon_{c}+\hbar \omega_{q}\right) \delta\left(\epsilon_{a}-\epsilon_{v}+\hbar \omega_{q^{\prime}}\right),
\end{aligned}
$$

where $b_{c}, b_{v}, m_{s}, m_{j}$ and $t$ are indexes for conduction and valence bands, spin, magnetic quantum number and an atom in the unit cell. Here $t$ summation includes those atoms in the unit cell which have the core state of the specified energy $\epsilon_{a}$. Using the conventional LMTO notation [10] the matrix element has the form 11]

$$
M_{t, m_{j}, m_{s}}^{b, \vec{k}}=\sum_{l, m} i^{l}\left(A_{t, l, m}^{b, \vec{k}} M_{t, l}^{r}+B_{t, l, m}^{b, \vec{k}} \dot{M}_{t, l}^{r}\right) M_{m_{j}, l, m, m_{s}}^{a},
$$

where $l$ and $m$ are angular momentum and magnetic quantum numbers of the valence and conduction states. $M_{t, l}^{r}, \dot{M}_{t, l}^{r}$ and $M_{m_{j}, l, m, m_{s}}^{a}$ are radial matrix element, its energy derivative and angular matrix element, respectively, having the following properties.

$$
\begin{gathered}
M_{t, l}^{\mathrm{r}}=\int R_{t}(r) r \phi_{t, l}(r) r^{2} d r \\
\dot{M}_{t, l}^{\mathrm{r}}=\int R_{t}(r) r \dot{\phi}_{t, l}(r) r^{2} d r \\
M_{m_{j}, l, m, m_{\mathrm{s}}}^{\mathrm{a}}=<j, m_{j}|\hat{\epsilon} \cdot \hat{r}| l, m, m_{\mathrm{s}}>,
\end{gathered}
$$

where $R_{t}(r)$ is the radial part of the core-level wave function and $\phi_{t, l}(r)$ is that of the valence or conduction band, $\hat{\epsilon}$ is the polarization vector of the photon, $j$ refers to the total angular momentum of the core state and $<j, m_{j} \mid$ and $\mid l, m, m_{\mathrm{s}}>$ are the angular parts of the core and valence/conduction states, respectively. The angular matrix element leads to the selection rules between the initial and final electronic states in the absorption and emission of a photon in the scattering process $(\Delta l= \pm 1)$. The 
localized core electron state of an atom $t$ centered at a point $\vec{r}_{\mathrm{a}}$ is

$$
\phi_{t, m_{j}}^{\mathrm{a}}(\vec{r})=R_{t}\left(\left|\vec{r}-\overrightarrow{\mathrm{r}_{\mathrm{a}}}\right|\right) \mid j, m_{j}>.
$$

$A_{t, l, m}^{b \vec{k}}$ and $B_{t, l, m}^{b \vec{k}}$ are coefficients which depend on the crystal structure and the potential of the investigated system. The wave function of the valence and conduction electrons has the form

$$
\Psi^{b \vec{k}}(\vec{r})=\sum_{t, l, m} i^{l}\left(A_{t, l, m}^{b \vec{k}} \phi_{t l}(r)+B_{t, l, m}^{b \vec{k}} \dot{\phi}_{t l}(r)\right) \mid l, m, m_{\mathrm{s}}>
$$

where $\phi_{t l}(r)$ and $\dot{\phi}_{t l}(r)$ are the partial wave and its energy derivative. LMTO wave functions are linearized with respect to energy at some suitable fixed energy $E_{\nu l}$. Because we are interested in energy eigenvalues $E_{b \vec{k}}$ near to $E_{\nu l}$ we can substitute $B_{t, l, m}^{b \vec{k}}$ coefficients by their approximate form $A_{t, l, m}^{b \vec{k}}\left(E_{b \vec{k}}-E_{\nu l}\right)$, which reduces the required memory in computations about a factor of two.

The electronic structure calculations were performed using the scalar-relativistic tight-binding linear muffintin orbital method in the atomic sphere approximation (ASA) 12]. The valence states consisted of $\mathrm{Mg} 3 s, 3 p$ and $3 d$ states and B $2 s, 2 p$ and $3 d$ states. The hexagonal unit cell contained three atoms and three empty spheres. We used lattice parameters $a=b=2.99 \AA$ and $c=3.41 \AA$ corresponding to equilibrium volume in our calculations. For the exchange-correlation potential the parametrized form by Perdew and Zunger 13] was used. The number of $\vec{k}$ points was 648 in the whole Brillouin zone.

The geometry used in the calculations is the following. The direction of the absorbed photon makes an angle $\theta$ with respect to the hexagonal $c$-axis of the $\mathrm{MgB}_{2}$ single crystal. The absorbed photon is linearly polarized and the polarization is in the plane containing the direction of the photon and the $c$-axis of the crystal. In this way the component of the polarization vector of the absorbed photon along the $c$-axis can be varied from 0 to 1 by increasing $\theta$ from $0^{\circ}$ to $90^{\circ}$. The direction of the emitted photon is perpendicular to the direction of the absorbed photon and makes a $90^{\circ}-\theta$ angle with respect to the $c$-axis. For the emitted photon we have used two different linear polarizations. The polarization is either in the $(a, b)$-plane or along the direction of the absorbed photon. All our results are expressed using an energy scale where the Fermi energy is $0 \mathrm{eV}$.

Overall features of both XES and XAS of $\mathrm{MgB}_{2}$ are well produced by band structure calculations [14]. Thus RIXS experiments are also expected to be explained within the ordinary band structure picture. Because the valence hole is delocalized and the 1s core hole in boron is well screened [15] it is reasonable to use ground state orbitals to obtain theoretical spectra. Due to the conservation of both energy and momentum we need detailed band structure data in order to interpret the spectra. The calculated band structure is shown in Figure1 Since we are interested in absorption and emission of photons

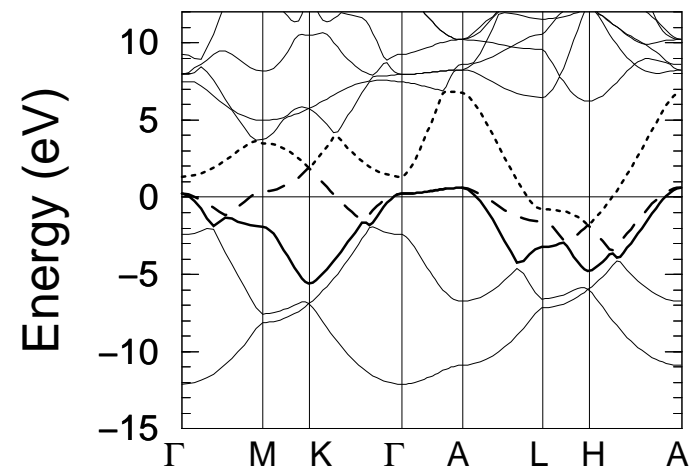

FIG. 1: Band structure of $\mathrm{MgB}_{2}$. Bands 3, 4 and 5, which cross the Fermi level, are shown by a thicker line: solid, dashed and dotted, respectively.

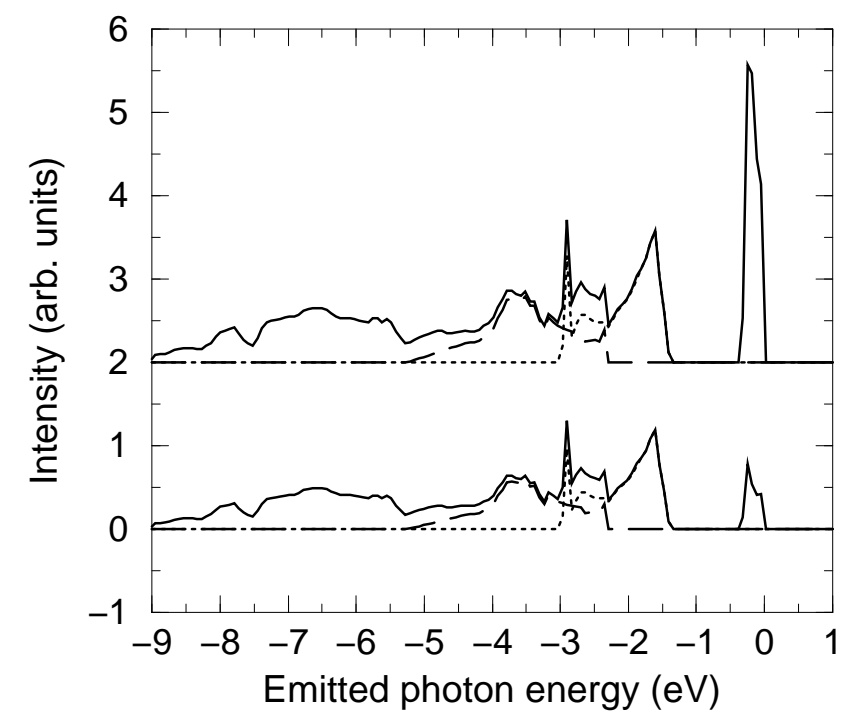

FIG. 2: RIXS spectra calculated at $\theta=60^{\circ}$. Excitation energy is $0.2 \mathrm{eV}$ relative to the Fermi energy. Polarization of the emitted photon is in the $(a, b)$-plane (upper spectrum) and in the direction of the absorbed photon (lower spectrum). Dashed and dotted lines correspond to spectra calculated using bands $(3,5)$ and $(4,5)$, respectively.

coupled with electronic states near to the Fermi energy, the most relevant bands are those which cross the Fermi level, i.e. bands number 3-5. Zhang et al. [16] have measured RIXS spectra at different angles of incidence $(\theta=$ $15^{\circ}, 45^{\circ}, 60^{\circ}$ with respect to the $c$-axis of the $\mathrm{MgB}_{2}$ crystal) and using different excitation energies (187.25 eV $188.25 \mathrm{eV}$, Fermi energy: $\left.E_{F}=187.28 \mathrm{eV}\right)$. They found two peaks in their spectra. The intensity of the peak just below the Fermi energy depends clearly on both direction and energy of the exciting radiation. On the other hand, the intensity of the peak at about $2 \mathrm{eV}$ below the Fermi energy was considered to be unchanging.

In Figure 2 we show our results for the $\theta=60^{\circ}$ incidence in the case of two polarizations of the emitted photon. The unpolarized emission spectrum is an average of these two extreme cases. The peak just below the 


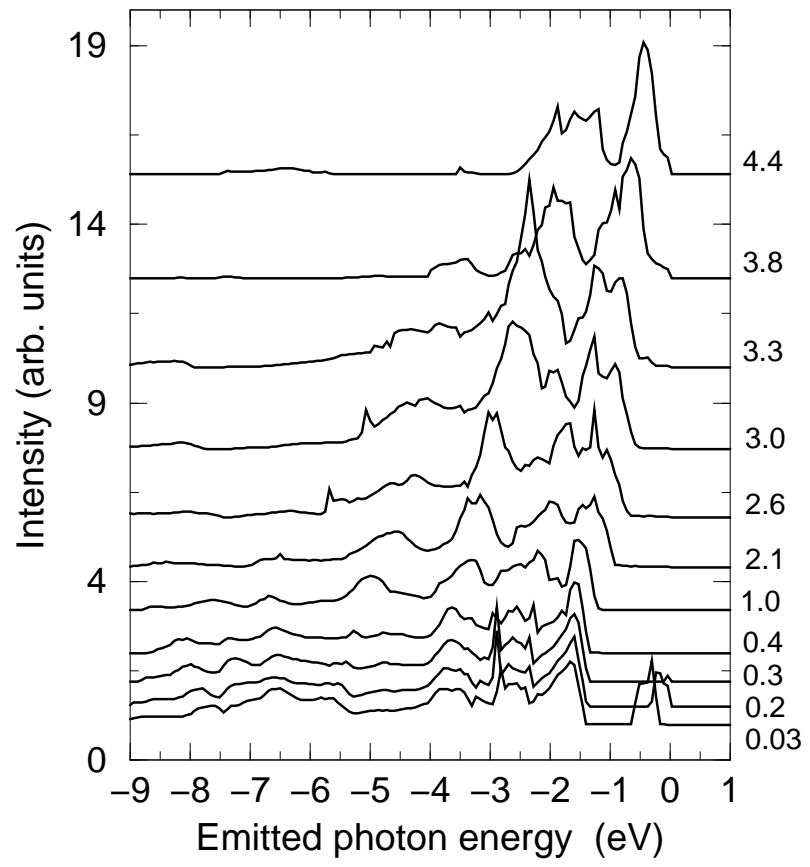

FIG. 3: RIXS spectra calculated at $\theta=75^{\circ}$. Exitation energy (in eV), relative to the Fermi energy, is shown beside each spectrum. Polarization of the emitted photon is in the $(a, b)$-plane.

Fermi energy is solely due to absorption to the band 4 and emission from the band 3 . The features between -4 $\mathrm{eV}$ and $-1.5 \mathrm{eV}$ consist almost entirely from RIXS involving band pairs $(3,5)$ and $(4,5)$. By increasing angle $\theta$ one can measure more directly excitations to the $\pi$ band.

In Figure 3 we show the spectra corresponding to $\theta=75^{\circ}$ and the polarization vector of the emitted radiation, in our calculation, being in the $a b$-plane. Near the top of the valence band there appears a peak which disappears when the excitation reaches $0.4 \mathrm{eV}$ above the Fermi energy. The broad structure from $-4 \mathrm{eV}$ to -1.5 eV shows not much dispersion up to excitation energies $0.4 \mathrm{eV}$ above the Fermi energy. However, using excitation energies above $1 \mathrm{eV}$ the intensity of the peaks increases considerably and the position of the peaks shifts about $1 \mathrm{eV}$ closer to the Fermi level as the excitation energy increases. To decide what bands and what parts of the Brillouin zone are responsible for each peak in the spectrum we have calculated some of the spectra band by band. As Figure 4 shows the peak around $-2 \mathrm{eV}$ (low excitation energies) is mainly due to transitions involv- ing bands 4 and 5 . In the same way, the peak around $-3.5 \mathrm{eV}$ is mainly due to transitions involving bands 3 and 5. From Figure 1we see that both of these peaks are produced close to the $\mathrm{L}-\mathrm{H}$ line in the Brillouin zone. In this region, near the Fermi energy, the constant energy surfaces of the antibonding $\pi$ band have a tubular form [17].

In summary, we have calculated RIXS spectra of $\mathrm{MgB}_{2}$ using Kramers-Heisenberg formula with TB-

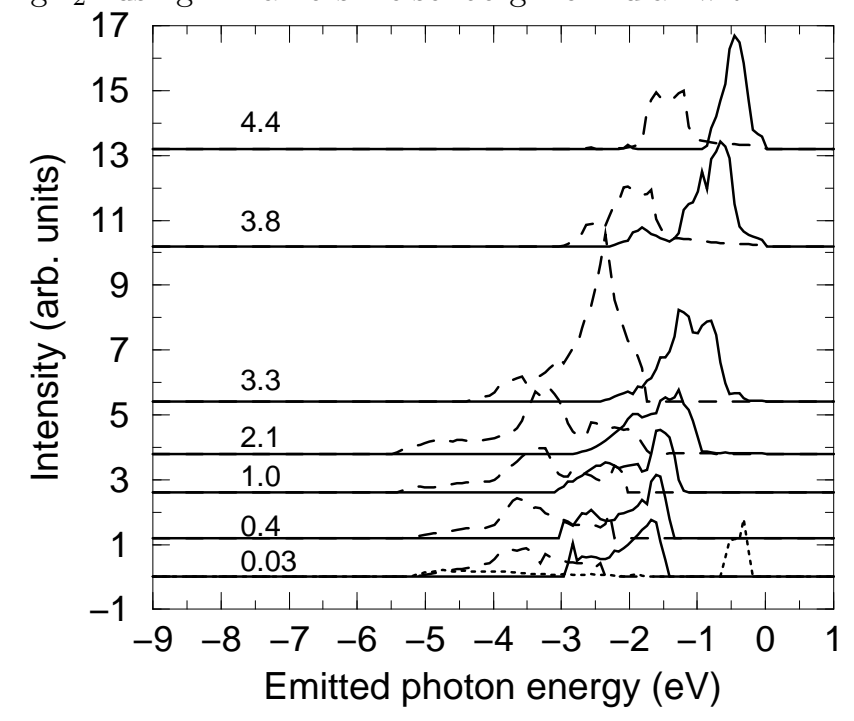

FIG. 4: Some of the spectra shown in Fig. 3 calculated using the bands $(3,4)$ (dotted), (3,5) (dashed) and $(4,5)$ (solid).

LMTO eigenvalues and eigenstates for occupied and unoccupied electronic states. The results presented show that $\mathrm{MgB}_{2}$ can be analysed and interpreted on the basis of ground state band structure calculations. In addition we predict nonlinear emission structures related with the excitation of $1 \mathrm{~s}$ electrons to the unoccupied antibonding $\pi$ band in the neighbourhood of the $\mathrm{L}-\mathrm{H}$ line in the Brillouin zone.

We acknowledge computer resources of CSC - Scientific Computing Ltd., Espoo, Finland. This work has been in part supported by the Academy of Finland, Grant No. 51583 (K.K. and V.K.) and the Turku University Foundation (K.K. and W.H.).

*Author to whom correspondence should be addressed. Electronic address: kokko@utu.fi
[1] J. Akimitsu, Symposium on Transition Metal Oxides, Sendai, January 10, 2001.

[2] J. Nagamatsu, N. Nakagawa, T. Muranaka, Y. Zenitani and J. Akimitsu, Nature (London) 410, 63 (2001).

[3] R.P. Vasquez, C.U. Jung, Min-Seok Park, Heon-Jung
Kim, J.Y. Kim, and Sung-Ik Lee, Phys. Rev.B, 64, 052510 (2001).

[4] H. Uchiyama, K.M. Shen, S. Lee, A. Damascelli, D.H. Lu, D.L. Feng, Z.-X. Shen, and S. Tajima, Phys. Rev. Lett. 88, 157002 (2002). 
[5] E.Z. Kurmaev, I.I. Lyakhovskaya, J. Kortus, A. Moewes, N. Miyata, M. Demeter, M. Neumann, M. Yanagihara, M. Watanabe, T. Muranaka, and J. Akimitsu, Phys Rev. B 65, 134509 (2002).

[6] A. Kotani and S. Shin, Rev. Mod. Phys. 73, 203 (2001).

[7] J. A. Carlisle, E. L. Shirley, L. J. Terminello, J. J. Jia, T. A. Callcott, D. L. Ederer, R. C. C. Perera, and F. J. Himpsel, Phys. Rev. B 59, 7433 (1999).

[8] Y. Ma, Phys. Rev. B 49, 5799 (1994); P. D. Johnson and Y. Ma, Phys. Rev. B 49, 5024.

[9] W. Hergert, K. Kokko, and R. Laihia (unpublished).

[10] H. L. Skriver, The LMTO Method edited by M. Cardona and P. Fulde, Springer Series in Solid-State Sciences Vol. 41 (Springer, Berlin, 1984).

[11] R. Laihia, K. Kokko, W. Hergert, and J.A. Leiro, Phys. Rev. B 58, 1272 (1998).

[12] O. K. Andersen, O. Jepsen, and D. Glötzel, Proc. Int. School of Physics 'Enrico Fermi' (Course LXXXIX)
Highlights of Condensed-Matter Theory, edited by F. Bassani, F. Fumi, and M. P. Tosi, (North-Holland, Amsterdam, 1985), p. 59.

[13] J. P. Perdew and A. Zunger, Phys. Rev. B 23, 5048 (1981).

[14] J. Nakamura, N. Yamada, K. Kuroki, T.A. Callcott, D.L. Ederer, J.D. Denlinger, and R.C.C. Perera, Phys. Rev. B 64, 174504/1-4 (2001).

[15] K. Kokko, V. Kulmala, and J. A. Leiro, Phys. Rev. B 66, $165114(2002)$

[16] G.P. Zhang, G.S. Chang, T.A. Callcott, D.L. Ederer, W.N. Kang, Eun-Mi Choi, Hyeong-Jin Kim, and Sung-Ik Lee, cond-mat/0302466

[17] J. Kortus, I.I. Mazin, K.D. Belashchenko, V.P. Antropov, and L.L. Boyer, Phys. Rev. Lett. 86, 4656 (2001). See Fig. 3 in their article, where the antibonding $p_{z}$ band gives the red tubular part of the Fermi surface around the $\mathrm{L}-\mathrm{H}$ line. 